\title{
Cerebral sinovenous thrombosis in children: clinical presentation, locations, and acquired and inherited prothrombotic risk factors
}

\author{
Yeter Düzenli Kar ${ }^{1,2}$, Zeynep Canan Özdemir ${ }^{2 \odot}$, Kürşat Bora Çarman ${ }^{3 \odot, ~}$ \\ Coşkun Yarar ${ }^{3 \oplus}$, Neslihan Tekin ${ }^{4 \oplus}$, Özcan Bör ${ }^{2 \oplus}$ \\ ${ }^{1}$ Division of Pediatric Hematology and Oncology, Department of Pediatrics, Afyonkarahisar Health Sciences University, \\ Afyonkarahisar; Divisions of ${ }^{2}$ Pediatric Hematology and Oncology, ${ }^{3}$ Pediatric Neurology and ${ }^{4}$ Neonatology, Department of Pediatrics, \\ Eskişehir Osmangazi University Faculty of Medicine, Eskişehir, Turkey.
}

\begin{abstract}
Background. Cerebral sinovenous thrombosis (CSVT) in children is a rare and life-threatening cerebrovascular disease. Hence, we evaluated its clinical presentations, inherited and acquired prothrombotic risk factors along with the accompanying diseases, the thrombosis locations as well as the outcomes of anticoagulant therapy in children with CSVT.

Methods. The medical records of pediatric CSVT patients treated between January 2011 and September 2018 were analyzed retrospectively.

Results. The study included 29 children, 15 boys (51.7\%) and 14 girls (48.3\%), with the median age being 11 years (range:3 days-17 years). The most commonly presented complaint in neonates was seizures and in the non-neonatal age groups was a headache. Also, at least one acquired and/or inherited thrombophilic risk factor was identified in $89.7 \%$ of the patients. The most commonly acquired prothrombotic risk factors along with the accompanying diseases included infections, central venous catheter, and dehydration, while the most commonly inherited thrombophilic risk factors included heterozygous factor-V Leiden mutation and elevated lipoprotein (a). The most common thrombosis location was found to be the transverse sinus. Also, none of the patients died due to the thrombotic episode. Complications included epilepsy in five patients, hydrocephalus in one patient, and intracranial hypertension in another patient.
\end{abstract}

Conclusions. Clinicians need to be well aware of the inherited and acquired prothrombotic risk factors in CSVT. It should also be kept in mind that at-risk patients may also present with nonspecific signs and symptoms with no apparent neurological manifestation. The risk of acute complications and long-term sequelae can be substantially reduced if diagnosed early and initiated with appropriate treatment at the early stages.

Key words: cerebral sinovenous thrombosis, children, risk factors.

Cerebral sinovenous thrombosis (CSVT) in children is a rare and life-threatening cerebrovascular condition, and with increased clinical awareness and improved neuroradiological techniques, the disease can now be diagnosed more frequently and at earlier stages. Early diagnosis of CSVT is

$\triangle$ Yeter Düzenli Kar

yeterduzenli@yahoo.com

10th February 2021, revised 12th April 2021,

11th May 2021, accepted 21st May 2021. crucial since the risk of acute complications and long-term sequelae can be substantially reduced if appropriate treatment is initiated within the first few hours. ${ }^{1}$ The incidence of childhood CSVT occurs between 0.4 and 0.7 children per 100,000 children per year. ${ }^{2}$ The incidence is higher among neonates (30-50\%) compared to other pediatric age groups. There is a male predominance with boys accounting for approximately two-thirds of all cases in children. ${ }^{3}$ 
The etiology of childhood CSVT is multifactorial. Also, $33 \%$ to $99 \%$ of patients have inherited or acquired prothrombotic risk factors. The most well-known acquired prothrombotic risk factors along with the accompanying diseases include catheters, infection, dehydration, chronic inflammatory diseases, nephrotic syndrome, and malignancies. ${ }^{4}$ Studies have reported inherited thrombophilic risk factors including antithrombin deficiency, protein $\mathrm{C}$ and protein $\mathrm{S}$ deficiency, Factor $\mathrm{V}$ Leiden and prothrombin G20210A mutations, hyperhomocysteinemia, elevated circulating levels of factors II, VIII, IX, XI, and fibrinogen ${ }^{5}$, and high lipoprotein (a) level. ${ }^{6,7}$ Recent studies have indicated that mutations in the heterozygous methylenetetrahydrofolate reductase (MTHFR) gene alone cannot increase the risk of thrombosis. Hyperhomocysteinemia occurs in cases with homozygous MTHFR mutation due to the remethylation of impaired homocysteine to methionine. Hyperhomocysteinemia is also considered a strong risk factor for thrombosis in children. ${ }^{8,9}$

The most commonly recommended treatment for CSVT without significant intracranial hemorrhage includes anticoagulation treatment initially with either unfractionated heparin (UFH) or low-molecular-weight heparin (LMWH) for neonates and children. This initial therapy is followed by LMWH for 6 weeks to 3 months in neonates and LMWH or oral anticoagulant therapy for 3 to 6 months in children. ${ }^{10}$ The present study evaluated the clinical presentations, inherited and acquired prothrombotic and accompanying diseases risk factors, thrombosis locations along with the results of anticoagulant therapy in CSVT patients treated in our hospital over the last 8 years.

\section{Material and Methods}

1. The medical records of pediatric patients with CSVT who underwent treatment and follow-up in the pediatric and neonatal intensive care units between January 2011 and
September 2018 were analyzed retrospectively. The study was approved by the local ethics committee of Eskişehir Osmangazi University Clinical Researches (09.10.2018, no:17). The patients' age, sex, inherited and acquired prothrombotic risk factors and accompanying diseases, neurological symptoms and findings, thrombosis locations, treatment, and outcomes were reviewed from their medical records.

CSVT was diagnosed using brain magnetic resonance imaging and/or brain magnetic resonance venography. The results of etiological investigations were analyzed in all patients which included the complete blood count, cholesterol, triglycerides, lipoprotein (a), protein C, protein S, D-dimer, fibrinogen, antithrombin, homocysteine, C-reactive protein levels, activated protein $\mathrm{C}$ resistance, prothrombin time, activated partial thromboplastin time, antinuclear antibody (ANA) and extractable nuclear antigen antibody (ENA) profiles, and antiphospholipid and anticardiolipin IgG antibodies. The genetic mutation analysis in Factor V Leiden G1691A, prothrombin G20210A mutation, MTHFR C677T, and MTHFR A1298C were evaluated.

\section{Statistical analysis}

Data were analyzed with SPSS 17.0 statistical software package (SPSS Inc., Chicago, NY, USA) using descriptive statistics. The ShapiroWilk test was used to check whether numerical variables were normally distributed while the Chi-square $\left(\chi^{2}\right)$ test (exact method) was used to compare categorical variables.

\section{Results}

\section{Patient Population}

This study included a total of 29 children. Of which, five were neonates ( $0-28$ days); with two $(40 \%)$ females and three $(60 \%)$ male neonates. The non-neonatal age groups (29 days- 18 years) included 12 girls (50\%) and 12 boys (50\%) with a median age of 11 years (minimum 3 days, maximum 17 years) (Fig. 1). 


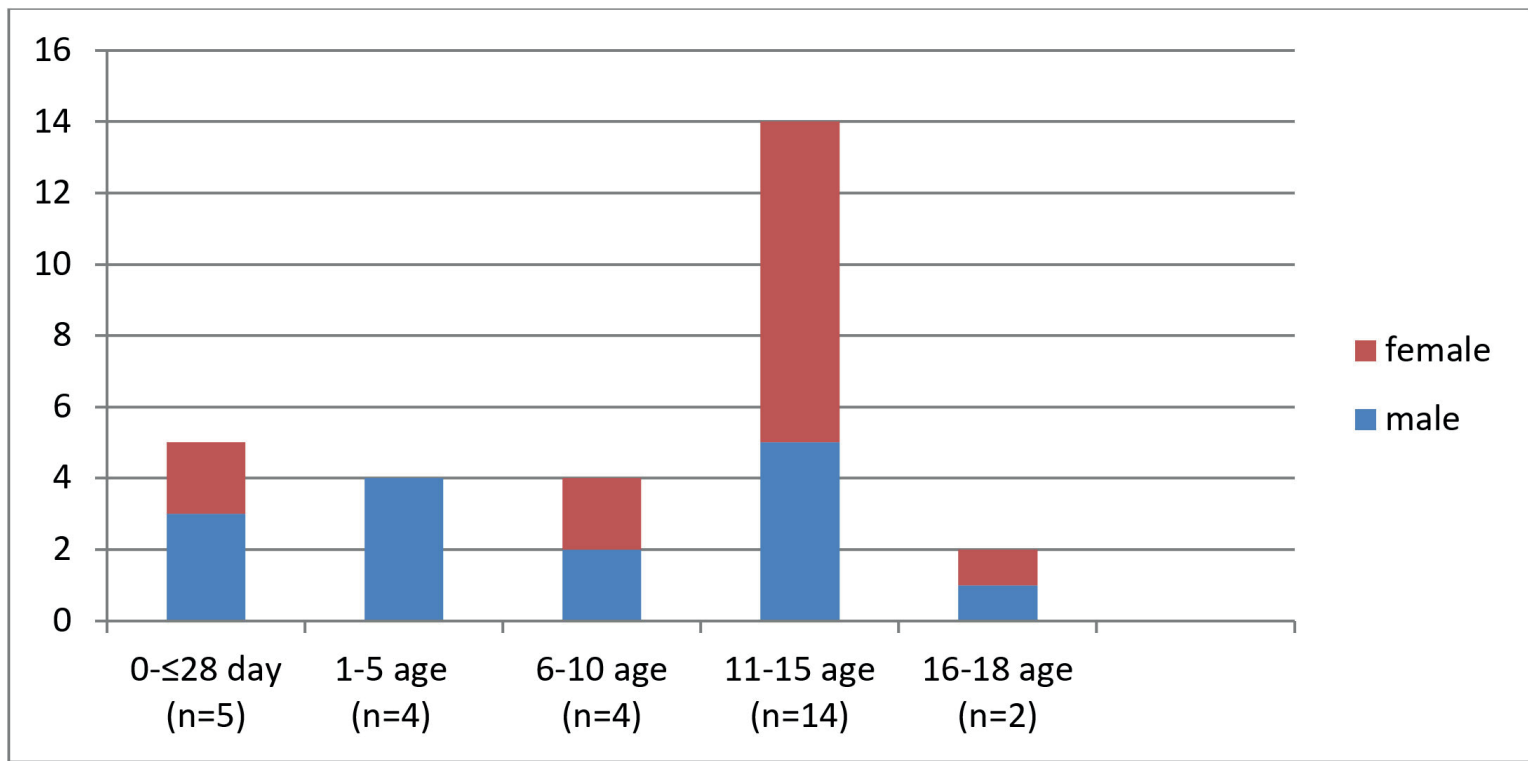

Fig. 1. Sex distribution of pediatric cerebral sinovenous thrombosis patients according to age group.

\section{Clinical presentation (Neurological symptoms and findings)}

All five neonates with CSVT presented with seizures $(100 \%)$, accompanied by hemiparesis in two patients $(40 \%)$, change in consciousness in two $(40 \%)$ patients, and respiratory failure in one $(20 \%)$ of the patients. Patients in the nonneonatal age groups presented with a headache (87.5\%), vertigo (50\%), diplopia (29.2\%), papilledema $(29.2 \%)$, hemiparesis $(29.2 \%)$, change in consciousness $(25 \%)$, seizures $(20.8 \%)$, facial paralysis $(12.5 \%)$, ptosis $(8.3 \%)$, and numbness $(8.3 \%)$ (Table I).

\section{Acquired and inherited thrombophilic risk factors}

At least one acquired and/or inherited thrombophilic risk factor was identified in 26 patients $(89.7 \%)$, while the other three patients $(10.3 \%)$ showed no known risk factors. Seven of the 21 patients with acquired risk factors also had inherited thrombophilic risk factors. The most commonly acquired risk factors included infections $(24.1 \%)$, a central venous catheter $(6.9 \%)$, and dehydration $(6.9 \%)$, while the most commonly inherited thrombophilic risk
Table I. Distribution of the patients age, sex, and neurological symptoms and findings.

\begin{tabular}{lcc}
\hline Neonates (0-28 days) & $\mathrm{n}$ & $\%$ \\
\hline Seizures & 5 & 100 \\
Change in consciousness & 2 & 40 \\
Hemiparesis & 2 & 40 \\
Respiratory failure & 1 & 20 \\
\hline Infants, children, and adolescents & & \\
(29 days-18 years) & & \\
\hline Headache & 21 & 87.5 \\
Vertigo & 12 & 50 \\
Hemiparesis & 7 & 29.2 \\
Double vision & 7 & 29.2 \\
Papilledema & 7 & 29.2 \\
Change in consciousness & 6 & 25.0 \\
Seizures & 5 & 20.8 \\
Facial paralysis & 3 & 12.5 \\
Ptosis & 2 & 8.3 \\
Numbness & 2 & 8.3 \\
\hline
\end{tabular}

factors included heterozygous factor V Leiden mutation (13.8\%) and elevated lipoprotein (a) level (13.8\%) (Table II). All five of the neonates had an acquired risk factor and accompanying diseases (dehydration in two, severe infection [sepsis] in two, and ventriculoperitoneal shunt 
Table II. Acquired and inherited thrombophilic risk factors of the patients.

\begin{tabular}{|c|c|}
\hline Risk Factors & $\mathrm{n}(\%)$ \\
\hline Acquired Prothrombotic Risk Factors & $21(72.4)$ \\
\hline Infection $(4)^{*}$ & $7(24.1)$ \\
\hline Jugular venous catheter & $2(6.9)$ \\
\hline Dehydration $(1)^{\neq}$ & $2(6.9)$ \\
\hline Obesity & $2(6.9)$ \\
\hline Chronic kidney disease + oxalosis $(1)^{\mu}$ & $1(3.5)$ \\
\hline Juvenile idiopathic arthritis $(1)^{\infty}$ & $1(3.5)$ \\
\hline Nephrotic syndrome & $1(3.5)$ \\
\hline Mental retardation + epilepsy & $1(3.5)$ \\
\hline Congenital heart disease & $1(3.5)$ \\
\hline Acute leukemia & $1(3.5)$ \\
\hline Hereditary spherocytosis & $1(3.5)$ \\
\hline Hydrocephalus + ventriculoperitoneal shunt & $1(3.5)$ \\
\hline Inherited Thrombophilic Risk Factors & $13(44.8)$ \\
\hline Factor V Leiden mutation (heterozygous) & $4(13.8)$ \\
\hline Lipoprotein(a) elevation (>30 mg/dL) & $4(13.8)$ \\
\hline Prothrombin G20210AI mutation (heterozygous) & $2(6.9)$ \\
\hline Hyperhomocysteinemia + homozygous/compound heterozygous MTHFR & $3(10.3)$ \\
\hline No known risk factors & $3(10.3)$ \\
\hline
\end{tabular}

*Accompanied by heterozygous factor V Leiden mutation in 2 patients, lipoprotein(a) elevation in 1 patient, hyperhomocysteinemia + homozygous/compound heterozygous MTHFR in 1 patient.

${ }^{*}$ Accompanied by lipoprotein(a) elevation in 1 patient.

${ }^{\mu}$ Accompanied by heterozygous factor V Leiden mutation and hyperhomocysteinemia + homozygous/compound heterozygous MTHFR in 1 patient.

${ }^{\infty}$ Accompanied by heterozygous prothrombin G20210AI mutation in 1 patient.

and hydrocephalus in one neonate). One of the neonates with dehydration also had elevated lipoprotein (a) level as an associated inherited risk factor. However, no inherited risk factors were identified in the other neonates.

Of the two neonates with infection as an acquired risk factor, one developed transverse and sigmoid sinus thrombosis following sepsis, while the other developed cerebral and Galen vein thrombosis after sepsis. Cases associated with infection in the other age groups included three patients developing lateral sinus thrombosis after otitis and mastoiditis, one developing cavernous sinus thrombosis after periorbital cellulitis, and the other developing bilateral transverse sinus thrombosis after meningitis.

\section{Thrombotic Locations}

Of the CSVT patients in this study, $44.8 \%$ had thrombosis in a single anatomic venous system, while $55.2 \%$ showed involvement of multiple venous systems. The most common anatomic site of thrombosis was the transverse sinus (Table III). None of our patients had thrombosis with intracranial hemorrhage.

\section{Treatment}

Treatment was initiated with unfractionated heparin or LMWH in all patients. LMWH was preferred over UFH due to its ease of administration, particularly in newborns and infants, patients with high hemorrhage risk, and the one for whom continuous vascular access was not possible. ${ }^{8}$ 
Table III. Thrombosis locations of patients diagnosed with central sinovenous thrombosis.

\begin{tabular}{lc}
\hline Thrombosis Location & $\mathrm{n}(\%)$ \\
\hline Single anatomic venous system & $13(44.8)$ \\
Transverse sinus* & $9(31.0)$ \\
Sagittal sinus & $1(3.5)$ \\
Jugular vein thrombus & $1(3.5)$ \\
Cavernous sinus & $1(3.5)$ \\
Sphenoparietal sinus & $1(3.5)$ \\
Multiple anatomic venous systems & $16(55.2)$ \\
Transverse sinus + Sigmoid sinus & \\
Bilateral transverse sinus & $5(17.2)$ \\
Bilateral sagittal sinus & $3(10.3)$ \\
Sinus rectus + Inferior sagittal sinus & $1(3.5)$ \\
Sinus rectus + sinus confluence & $1(3.5)$ \\
Transverse sinus + Galen vein & $\alpha$ \\
Sagittal vein + Transverse sinus & $1(3.5)$ \\
Cerebral vein + Galen vein & \\
Jugular vein + Transverse sinus & $1(3.5)$ \\
Transverse sinus + Sigmoid sinus + Jugular vein & $1(3.5)$ \\
\hline
\end{tabular}

${ }^{*} 1$ neonate, ${ }^{\neq} 2$ neonates, ${ }^{\alpha} 1$ neonate,${ }^{\beta} 1$ neonate.

All neonates received anticoagulant therapy of LMWH for 3 months. In three non-neonatal patients, anticoagulation was initiated with UFH for the first 5 to 7 days, which was then continued with an oral anticoagulant (warfarin) administration. Twenty-one patients started treatment with $\mathrm{LMWH}$, where 15 continued treatment with LMWH while six of them were switched to oral anticoagulant (warfarin) after 5 to 7 days. All patients were treated for at least 3 to 6 months. None of the patients had anticoagulant-related bleeding complications.

\section{Outcomes}

The mean follow-up time was 24 months. None of the patients died due to their thrombotic episodes. Five patients developed epilepsy, one patient developed hydrocephalus, and one patient developed intracranial hypertension. Two epilepsy patients and one hydrocephalus patient were neonates while the non-neonatal age group showed other complications. One patient's ptosis resolved after about one year.

\section{Discussion}

CSVT is a rare cerebrovascular disease that can occur in children of any age starting from the neonatal period, and carries a high risk of mortality and neurological sequelae. It is observed more commonly in males (56-75\%). ${ }^{6,8,11-}$ ${ }^{16}$ In the present study, male predominance was observed in the neonatal group consistent with the literature, whereas males and females were equally represented in the other age groups. The incidence of CSVT is reported to be highest in the neonatal period. ${ }^{6,11,14,17-20}$ Beside the neonatal period, it was more common in the 6-11 year age group (36.84\%) and in the 15-18 age group patients $(35.29 \%) .{ }^{15,21}$

CSVT patients can exhibit various signs and symptoms such as headaches, seizures, papilledema, cranial nerve palsies, motor weakness, and altered mental status. The most common neurological symptoms and findings in the literature include seizures in neonates ${ }^{4}$, whereas the headache was reported in $90 \%$ of 
adults and $60 \%$ of children. ${ }^{22}$ All the neonates in this study presented with seizures. The most common neurological symptoms and findings in the non-neonatal age groups were headache $(87.5 \%)$, dizziness $(50 \%)$, hemiparesis $(29.2 \%)$, and seizures $(20.8 \%)$. Less frequent findings were diplopia, papilledema, numbness, ptosis, and facial paralysis. Although seizures were more common in neonates, they may also occur in any child with CSVT. In addition to seizures, two of our neonates showed changes in consciousness, two showed hemiparesis, while one developed respiratory failure. The incidence of seizures in all childhood CSVT was reported as $26.9 \%$ by Lolli et al. ${ }^{20}, 34.8 \%$ by Ozcan et al. ${ }^{5}, 37.5 \%$ by Javed et al. ${ }^{16}, 37.9 \%$ by Heller et al. ${ }^{11}$ and $40 \%$ by Sèbire et al. $^{14}$. Hemiparesis was reported at a rate of 13$37.5 \%$ in the literature, which was consistent with our findings. Alterations in mental status may have manifested as irritability, stupor, or coma. ${ }^{6,23,24}$ Clinically, seizures and coma were reported as poor prognostic factors ${ }^{23}$, while isolated headache was considered a favorable prognostic factor. ${ }^{24}$ Isolated headache was the most commonly presented complaint in most of our patients. Also, none of our patients went into a coma or died due to CSVT.

Acquired prothrombotic risk factors along with the accompanying diseases reported in the etiology of CSVT include infections, dehydration, surgery, jugular or subclavian central venous catheters, solid tumors, leukemia and lymphomas, anemia, autoimmune diseases, renal diseases, obesity, metabolic disorders, birth asphyxia, and cardiac malformations. ${ }^{13}$ In this study, $10.3 \%$ of the patients showed no known acquired prothrombotic risk factors and accompanying diseases or inherited risk factors, while the other $89.7 \%$ showed at least one acquired and/or inherited risk factor. Similar to our study, Wasay et al. ${ }^{19}$ and Lolli et al. ${ }^{20}$ reported that $90 \%$ and $84.3 \%$ of all childhood CSVT cases, respectively, indicated the presence of one or more acquired prothrombotic risk factors and/or inherited thrombophilic risk factors. Acquired prothrombotic risk factors were identified in $70.5 \%$ of patients in the study by Heller et al. ${ }^{11}, 77.4 \%$ by Carvalho et al. ${ }^{18}, 81.5 \%$ by Kenet et al. ${ }^{25}, 86.8 \%$ by Viera et al. ${ }^{12}, 88.8 \%$ by Suppiej et al. ${ }^{26}$, and $100 \%$ by Sèbire et al. ${ }^{14}$. The prevalence of acquired prothrombotic risk factors along with the accompanying diseases in the present study was $72.4 \%$, which is consistent with the literature. Infection was the most commonly acquired prothrombotic risk factor in our study. Most infection-associated cases occurred in patients with head and neck infections. ${ }^{6,20,22,27}$ Also, head and neck infections were most common in our patient series, with three out of seven cases associated with infection occurring after otitis and mastoiditis, while one case occurred after periorbital cellulitis, and one after meningitis.

In $44.8 \%$ of our patients, at least one inherited thrombophilic risk factor was identified In previous large-scale studies conducted in Turkey, inherited thrombophilic risk factors were reported in $30-54 \%$ of patients. ${ }^{28-30}$ According to the literature, factor $\mathrm{V}$ Leiden mutation is the most commonly detected inherited thrombophilic risk factor in venous thromboembolism..$^{5,21,28,29}$ In the present study, the most commonly inherited thrombophilic risk factors were factor $\mathrm{V}$ Leiden mutation $(13.8 \%)$ and elevated lipoprotein (a) level $(13.8 \%)$. According to a study by Heller et al. ${ }^{11}$, these were also the most commonly identified inherited thrombophilic risk factors. Populationbased studies have shown that homocysteine metabolism-related gene polymorphisms such as MTHFR C677T and MTHFR A1298C do not alone cause thrombosis but increase the risk of thrombosis and cardiovascular disease when accompanied by elevated plasma homocysteine levels. Particularly, two known polymorphisms of the MTFHR gene (C677T and A1298C) have been associated with high homocysteine levels. ${ }^{5}$ Homocysteine is an amino acid that is formed as an intermediate product while converting methionine to cysteine and requires Vitamin B for conversion to either methionine or cysteine in reactions. Both $B$ vitamin deficiencies $\left(B_{6}\right.$ folic acid, $B_{12}$ ) and mutations in the MTHFR 
gene plays a role in the conversion of folate to its active form and are risk factors for hyperhomocysteinemia. ${ }^{22,31}$ In our study, hyperhomocysteinemia with homozygous/ compound heterozygous MTHFR mutation was the third most commonly inherited thrombophilic risk factor, which was detected in $10.3 \%$ of the patients.

The involvement in more than one anatomical region (multiple venous sinuses) was reported as more common in pediatric CSVT cases, with the transverse sinus being the most frequently involved region., 519,32 Multiple venous sinus thrombosis $(55.2 \%)$ and transverse sinus thrombosis were also more common in the present study. Wasay et al. ${ }^{19}$ observed multiple venous sinus involvement in $74 \%$ of their patients, with transverse sinus involvement being the most common (73\%). Ozcan et al. ${ }^{5}$ also reported that multiple sinus involvement was more frequent and reported the transverse sinus as the most commonly involved region (69.6\%). Viera et al. ${ }^{12}$ reported the transverse sinus to be most commonly involved as well (67.9\%). In contrast, Lolli et al. ${ }^{20}$ reported that $40.7 \%$ of patients had multiple sinus involvement while thrombosis was most frequently located in the transverse sinus in neonates $(27.3 \%)$ and the cortical veins in older children (31.3\%). Superior sagittal sinus thrombosis was common in some studies, which was detected at frequencies of $100 \%$ by Javed et al. $^{16}, 62.4 \%$ by Heller et al. ${ }^{11}$, and $47.4 \%$ by Bonduel et al. ${ }^{15}$. Of the five neonates in our study, thrombosis was located in the transverse and sigmoid sinuses in two neonates; in one neonate, it was located in the transverse sinus thrombosis; in another neonate, the location was in transverse sinus and Galen vein, and cerebral vein and Galen vein in another neonate.

According to the scientific statement from the American Heart Association/American Stroke Association, anticoagulation is the main treatment except in the case of otogenic lateral sinus thrombosis. The type, dose, and route of the anticoagulant agent were selected based on the individual patient's circumstances. For patients with CSVT and hemorrhagic infarction, otitis media/mastoiditis, head trauma, or neurosurgery, a multidisciplinary approach for anticoagulation should be undertaken.

American College of Chest Physicians EvidenceBased Clinical Practice Guidelines recommends anticoagulation treatment initially with either UFH or LMWH and subsequently with LMWH or vitamin K antagonists (VKA) for a minimum of 3 months for children with CSVT without significant ICH. If CSVT occlusion or ongoing symptoms persist after the initial 3 months of therapy, further administration of anticoagulation was suggested for 3 more months. ${ }^{7}$ For CSVT patients with significant hemorrhage, the radiologic monitoring of the thrombosis was suggested for 5 to 7 days, and anticoagulation was suggested if thrombus extension was noted at that time or initial anticoagulation was suggested for children without hemorrhage in close follow-up.,10 However, to choose one of these two options, the multidisciplinary decision has to be considered. In children with CSVT and recurrent risk factors (e.g., nephrotic syndrome, L-asparaginase therapy), prophylactic anticoagulation was suggested at times of risk factor recurrence. Thrombolysis, thrombectomy, or surgical decompression may be required only in children with severe CSVT, where no improvement was observed after initial anticoagulant therapy. Also, supportive care and neuroprotective measures such as replacement of intravenous fluids, oxygenation, the elevation of the head of the bed to $30^{\circ}$, and treatment of seizures are important too. Children with CSVT should be followed up for increased intracranial pressure and papilledema. Repeated MRV venography is required during the follow-up therapy to decide the duration of anticoagulation. ${ }^{7}$

None of our patients had thrombosis with intracranial hemorrhage. Therefore, anticoagulation was initiated in all our patients immediately upon diagnosis. Also, studies in children have reported that LMWH and UFH are safe in children, where anticoagulant therapy reduces mortality, preserves cognitive 
functions, and lowers the rate of recurrent thrombosis. ${ }^{7,24}$

The limitations of our study were as follows: a small number of patients, a single-center center experience-based study, and the retrospective nature of the study.

In conclusion, CSVT in children is a rare and life-threatening condition. Early diagnosis is critical since the risk of acute complications and long-term sequelae can be significantly reduced with an appropriate treatment approach implemented in the early stage. Therefore, clinicians need to be well aware of the clinical picture of CSVT and it is important to keep in mind that these patients may not have neurological symptoms such as seizures, diplopia, papilledema, numbness, ptosis, or facial paralysis, and may only be presented with nonspecific symptoms and findings, such as headache, dizziness, fatigue, and respiratory failure. Also, thorough knowledge of the acquired prothrombotic risk factors along with the accompanying diseases and inherited prothrombotic risk factors facilitates the early diagnosis of patients at risk.

\section{Ethical approval}

The study was approved by the local ethics committee (09.10.2018, no:17).

\section{Author contribution}

The authors confirm contribution to the paper as follows: study conception and design: YDK, ZCÖ, ÖB; data collection: YDK, KBÇ, CY, NT; analysis and interpretation of results: $\mathrm{YDK}$, ZCÖ; draft manuscript preparation: YDK, ZCÖ, ÖB. All authors reviewed the results and approved the final version of the manuscript.

\section{Source of funding}

None.

\section{Conflict of interest}

No conflict of interest.

\section{REFERENCES}

1. Carducci C, Colafati GS, Figà-Talamanca L, et al. Cerebral sinovenous thrombosis (CSVT) in children: what the pediatric radiologists need to know. Radiol Med 2016; 121: 329-341. https://doi.org/10.1007/ s11547-016-0630-9

2. Ritchey Z, Hollatz AL, WeitzenkampD, et al. Pediatric cortical vein thrombosis: frequency and association with venous infarction. Stroke 2016; 47: 866-868. https://doi.org/10.1161/STROKEAHA.115.011291

3. Ichord RN, Benedict SL, Chan AK, Kirkham FJ, Nowak-Göttl U; International Paediatric Stroke Study Group. Paediatric cerebral sinovenous thrombosis: findings of the International Paediatric Stroke Study. Arch Dis Child 2015; 100: 174-179. https://doi.org/10.1136/archdischild-2014-306382

4. Bektaş Ö, Teber S, Akar N, et al. Cerebral sinovenous thrombosis in children and neonates: clinical experience, laboratory, treatment, and outcome. Clin Appl Thromb Hemost 2015; 21: 777-782. https:// doi.org/10.1177/1076029614523491

5. Ozcan A, Canpolat M, Doganay S, et al. Cerebral sinus venous thrombosis and prothrombotic risk factors in children: a single- center experience from Turkey. J Pediatr Hematol Oncol 2018; 40: e369-e372. https://doi.org/10.1097/MPH.0000000000001149

6. Ichord R. Cerebral sinovenous thrombosis. Front Pediatr 2017; 5: 163 . https://doi.org/10.3389/ fped.2017.00163

7. Ferriero DM, Fullerton HJ, Bernard TJ, et al; American Heart Association Stroke Council and Council on Cardiovascular and Stroke Nursing. Management of stroke in neonates and children: a scientific statement from the American Heart Association/American Stroke Association. Stroke 2019; 50: e51-e96. https:// doi.org/10.1161/STR.0000000000000183

8. Acharya SS, Sarangi SN. Disorders of coagulation. In: Lanzkowsky P, Lipton JM, Fish JD (eds). Lanzkowsky's Manual of Pediatric Hematology and Oncology (6th ed). New York: Academic Press, 2016: 279-333. https://doi.org/10.1016/B978-0-12-8013687.00015-6

9. Yang JY, Chan AK. Pediatric thrombophilia. Pediatr Clin North Am 2013; 60: 1443-1462. https://doi. org/10.1016/j.pcl.2013.09.004 
10. Monagle P, Chan AKC, Goldenberg NA, et al. Antithrombotic therapy in neonates and children: antithrombotic therapy and prevention of thrombosis, 9th ed: American College of Chest Physicians Evidence-Based Clinical Practice Guidelines. Chest 2012; 141(Suppl 2): e737S-e801S. https://doi.org/10.1378/chest.11-2308

11. Heller C, Heinecke A, Junker R, et al; Childhood Stroke Study Group. Cerebral venous thrombosis in children: a multifactorial origin. Circulation 2003; 108: 1362-1367. https://doi.org/10.1161/01. CIR.0000087598.05977.45

12. Viera JP, Luis C, Monteiro JP, et al. Cerebral sinovenous thrombosis in children: clinical presentation and extension, localization and recanalization of thrombosis. Eur J Paediatr Neurol 2010; 14: 80-85. https://doi.org/10.1016/j. ejpn.2008.12.004

13. Kenet G, Kirkham F, Niederstadt T, et al; European Thromboses Study Group. Risk factors for recurrent venous thromboembolism in the European collaborative paediatric database on cerebral venous thrombosis: a multicentre cohort study. Lancet Neurol 2007; 6: 595-603. https://doi.org/10.1016/ S1474-4422(07)70131-X

14. Sébire G, Tabarki B, Saunders DE, et al. Cerebral venous sinus thrombosis in children: risk factors, presentation, diagnosis and outcome. Brain 2005; 128: 477-489. https://doi.org/10.1093/brain/awh412

15. Bonduel M, Sciuccati G, Hepner M, et al. Arterial ischemic stroke and cerebral venous thrombosis in children: a 12-year Argentinean registry. Acta Haematol 2006; 115: 180-185. https://doi. org/10.1159/000090932

16. Javed I, Sultan T, Rehman ZU, Yaseen MR. Clinical spectrum and outcome of cerebral venous sinus thrombosis in children. J Coll Physicians Surg Pak 2018; 28: 390-393. https://doi.org/10.29271/ jcpsp.2018.05.390

17. deVeber G, Andrew M, Adams C, et al; Canadian Pediatric Ischemic Stroke Study Group. Cerebral sinovenous thrombosis in children. $\mathrm{N}$ Engl J Med 2001; 345: 417-423. https://doi.org/10.1056/ NEJM200108093450604

18. Carvalho KS, Bodensteiner JB, Connolly PJ, Garg BP. Cerebral venous thrombosis in children. J Child Neurol 2001; 16: 574-580. https://doi. org/10.1177/088307380101600807

19. Wasay M, Dai AI, Ansari M, Shaikh Z, Roach ES. Cerebral venous sinus thrombosis in children: a multicenter cohort from the United States. J Child Neurol 2008; 23: 26-31. https://doi. org/10.1177/0883073807307976
20. Lolli V, Molinari F, Pruvo JP, Soto Ares G. Radiological and clinical features of cerebral sinovenous thrombosis in newborns and older children. J Neuroradiol 2016; 43: 280-289. https://doi. org/10.1016/j.neurad.2015.12.001

21. Tuckuviene R, Christensen AL, Helgestad J, Johnsen SP, Kristensen SR. Paediatric arterial ischaemic stroke and cerebral sinovenous thrombosis in Denmark 1994-2006: a nationwide population-based study. Acta Paediatr 2011; 100: 543-549. https://doi. org/10.1111/j.1651-2227.2010.02100.x

22. Ünver O, Ekinci G, Kutlubay BI, et al. Evaluation of cases with cerebral thrombosis in children. Turk Pediatri Ars 2016; 51: 87-93. https://doi.org/10.5152/ TurkPediatriArs.2016.3660

23. Hedlund GL. Cerebral sinovenous thrombosis in pediatric practice. Pediatr Radiol 2013; 43: 173-188. https://doi.org/10.1007/s00247-012-2486-z

24. Dlamini N, Billinghurst L, Kirkham FJ. Cerebral venous sinus (sinovenous) thrombosis in children. Neurosurg Clin N Am 2010; 21: 511-527. https://doi. org/10.1016/j.nec.2010.03.006

25. Kenet G, Waldman D, Lubetsky A, et al. Paediatric cerebral sinus vein thrombosis. A multi-center, casecontrolled study. Thromb Haemost 2004; 92: 713718. https://doi.org/10.1160/TH04-03-0182

26. Suppiej A, Gentilomo C, Saracco P, et al; Stroke Working Group of the Italian Registry of Pediatric Thrombosis. Paediatric arterial ischaemic stroke and cerebral sinovenous thrombosis. First report from the Italian Registry of Pediatric Thrombosis (R. I. T. I., Registro Italiano Trombosi Infantili). Thromb Haemost 2015; 113: 1270-1277. https://doi. org/10.1160/TH14-05-0431

27. Grunt S, Wingeier K, Wehrli E, et al; Swiss Neuropaediatric Stroke Registry. Cerebral sinus venous thrombosis in Swiss children. Dev Med Child Neurol 2010; 52: 1145-1150. https://doi. org/10.1111/j.1469-8749.2010.03722.x

28. Günes AM, Baytan B, Günay U. The influence of risk factors in promoting thrombosis during childhood: the role of acquired factors. Pediatr Hematol Oncol 2006; 23: 399-410. https://doi. org/10.1080/08880010600646324

29. Oren H, Devecioğlu O, Ertem M, et al. Analysis of pediatric thrombotic patients in Turkey. Pediatr Hematol Oncol 2004; 21: 573-583. https://doi. org/10.1080/08880010490500935

30. Ozbek N, Alioglu B, Avci Z, et al. Incidence of and risk factors for childhood thrombosis: a single-center experience in Ankara, Turkey. Pediatr Hematol Oncol 2009; 26: 11-29. https://doi. org/10.1080/08880010802423969 
31. Park WC, Chang JH. Clinical implications of methylenetetrahydrofolate reductase mutations and plasma homocysteine levels in patients with thromboembolic occlusion. Vasc Specialist Int 2014; 30: 113-119. https://doi.org/10.5758/vsi.2014.30.4.113
32. Amlie-Lefond C. Evaluation and acute management of ischemic stroke in infants and children. Continuum (Minneap Minn) 2018; 24(1, Child Neurology): 150170. https://doi.org/10.1212/CON.0000000000000559 